\title{
Potential of Polycaprolactone Nanofiber Scaffold for Ex Vivo Expansion of Cord Blood-Derived CD34+ Hematopoietic Stem Cells
}

\author{
Seyed Hadi Mousavi ${ }^{1}$, Saeid Abroun ${ }^{2}$, Masoud Soleimani ${ }^{2}$ and Seyed Javad Mowla ${ }^{3}$ \\ ${ }^{1}$ Department of Hematology, School of Allied Medical Sciences, Tehran University of Medical Sciences, Tehrar \\ ${ }^{2}$ Department of Hematology, Tarbiat Modares University, Tehran, Iran \\ ${ }^{3}$ Department of Molecular Genetics, Tarbiat Modares University, Tehran, Iran
}

*Corresponding author: Saeid Abroun, Department of Hematology, Faculty of medical sciences, Tarbiat Modarres University, Tehran, Iran, Tel: +98-21-82884563, Fax: +98-21-82884507

\begin{abstract}
Background: An efficient and practical ex vivo expansion of cord blood hematopoietic stem cells as an alternative source of HSC transplantation is crucial in understanding the potential of HSC transplantation in treating or supportive therapy in a variety of hematologic and non-hematologic disorders. The aim of this study was an ex vivo expansion of cord blood hematopoietic stem cells in a novel threedimensional polycaprolactone nanofiber scaffold coated with collagen.
\end{abstract}

Methods: After 10-day cultured of cord blood CD34+ cells in 2-Dimensional and 3-Dimensional culture system, the evaluation was performed by qRT-PCR, flow cytometry and clonogenicity.

Results: 3-Dimensional Polycaprolactone nano-scaffold coated with collagen provided higher total nucleated cells (50-fold vs. 38-fold) and CD34+ cells (20-fold vs. 2.6-fold) $(p<0.05)$ and compared to 2-Dimensional cell culture and before expansion had higher expression of homing and self-renewal genes and for VLA-4, hTERT and BMI-1 genes were statically significant $(p=0.0001)$. The expression of myeloid markers in 3-Dimensional scaffold was significantly higher than the 2-Dimensional culture system $(p<0.05)$. The total colony in 2-Dimensional culture was lower than 3 -Dimensional culture medium $(p<0.05)$.

Conclusion: This study demonstrated the synergistic effect between the three-dimensionality of the scaffold and collagen as an extracellular matrix protein, and the potential of this 3-Dimensional Polycaprolactone nanofiber scaffold coated with collagen for ex vivo expansion of HSCs.

\section{Keywords}

Cord blood expansion, Hematopoietic stem cell expansion, Cord blood transplantation, Tissue engineering, 3D culture, Nanofiber, Polycaprolactone

\begin{abstract}
Abbreviations
3D: Three-dimensional; BM: Bone Marrow; CB: Cord Blood; CFU: Colony-Forming Unit; ECM: Extracellular Matrix; GVHD: Graft-Versus-Host Disease; HLA: Human Leukocyte Antigen; HSC; Hematopoietic Stem Cell; HSCT: Hematopoietic Stem Cell Transplantation; PCL: Polycaprolactone; PGs: Proteoglycans; SEM: Scanning Electron Microscope; TNCs: Total Nucleated Cells; UCB: Umbilical Cord Blood
\end{abstract}

\section{Introduction}

Not surprisingly, after first successful cord blood (CB) hematopoietic stem cell transplantation (HSCT) in 1988 [1] and some limitations of bone marrow (BM), as a classic source of HSCT such as, paucity of donors and lack a human leukocyte antigen (HLA) identical donors [2], the use of umbilical cord blood (UCB) hematopoietic stem cell (HSC) has been growing in last decades and has become an efficacious treatment source of HSC for patients who suffer from hematological and nonhematological malignancy disorders [3]. Regrettably, despite the notable benefits include the relative ease of collection, ready availability as cryopreserved units from CB banks, as well as strong clinical evidence

Citation: Mousavi SH, Abroun S, Soleimani M, Mowla SJ (2019) Potential of Polycaprolactone Nanofiber Scaffold for Ex Vivo Expansion of Cord Blood-Derived CD34+ Hematopoietic Stem Cells. Int J Stem Cell Res Ther 5:059. doi.org/10.23937/2469-570X/1410059

Accepted: February 05, 2019: Published: February 07, 2019

Copyright: (c) 2019 Mousavi SH, et al. This is an open-access article distributed under the terms of the Creative Commons Attribution License, which permits unrestricted use, distribution, and reproduction in any medium, provided the original author and source are credited. 
supporting the use of HLA incompatible transplants with low risk of graft-versus-host disease (GVHD), extensive applications of $C B$ is reduced [4]. The main restrictive factor to $C B$ application is the low cell dose obtainable for transplantation [5]. It is well documented that transplantation with lower cell number has undesirable effects in patients as well as led to increase graft failure. Obviously, patient outcomes have a close correlation with total nucleated cell dose (TNC) per kilogram (kg) of body weight transplanted [6]. Therefore, CBT Less success in adults and restricted to children.

There are various approaches such as expanded in liquid cultures, co-culture with mesenchymal stromal cells, genetic manipulation, bioreactors and nanofiber scaffolds to overcoming the issue of low TNC [79]. The inefficiency of some of the aforementioned methods is due to the lack of mimicking of the BM microenvironment. An appropriate understanding of the 3D network of the BM can help to improve this issue.

HSCs reside in a 3D complex microenvironment containing mesenchymal stromal cells (adipocytes, osteoblasts, and endothelial cells), growth factors, hormones and multiple components of the extracellular matrix (ECM). This specialized and controlled microenvironment, termed "Niche" that regulates the size of the HSC pool and controls the HSC fate [10].

Studies of the recent years confirmed that imitating the elements of the stem cell niche will facilitate producing sufficient cell number, maintain the capacity for self-renewal and controlled differentiation in cell culture medium. In the components of BM microenvironments, ECM has critical functions in biochemical and biomechanical cues that are amplified. Adhesiveness or communication between HSCs and cell adhesion molecule supply homing or retaining HSCs in the bone marrow niche are facilitated by these elements that in tight association with stromal cells, osteoblasts, and signaling molecules they produce $[11,12]$. In several studies confirmed the importance of topographical architecture of the ECM in regulating cell growth, survival, migration, differentiation, tissue reorganization, and matrix remodeling. The ECM is composed of two major classes of macromolecules: Proteoglycans (PGs) and fibrous proteins (collagens, elastins, fibronectins, and laminins). Collagen is the most abundant fibrous protein within the ECM that regulate cell adhesion and migration $[11,13]$.

Ex vivo expansion of cord blood hematopoietic stem cells in nano-fibers scaffolds that imitate bone marrow microenvironment an attractive approach for resolving this important problem, low cell dose.

According to aforementioned studies and due to the importance of the cell number of HSCs in transplantation, in the current study, we propose a 3D polycaprolactone $(\mathrm{PCL})$ nano-scaffold coated with collagen culture system for ex vivo expansion of cord blood-derived CD34+ cells.

\section{Materials and Methods}

\section{Preparation and characterization the scaffolds}

Nanofibrous PCL scaffolds were fabricated by an electrospinning instrument. PCL ( $\mathrm{Mw}=80,000$; Sigma, Steinheim, Germany) was dissolved in chloroform $(9 \% \mathrm{w} / \mathrm{v})$ and added directly to dimethylformamide (Sigma, Steinheim, Germany) solution (10:1). Random nanofibrous scaffolds were obtained using low (100 rpm) speed rotating the disk.

PCL scaffolds sterilized by immersion in $70 \%$ ethanol for $1 \mathrm{~h}$, washed with PBS and then dried overnight under sterile condition. Scaffolds were located at 24well polystyrene plates and coated with collagen type I in $50 \mu \mathrm{g} / \mathrm{ml}$ concentration at the $40^{\circ} \mathrm{C}$ for $24 \mathrm{~h}$. Finally, we removed collagen solution and seeded CD34+ cells.

For scanning electron microscopy (SEM), the CD34+cells were washed with PBS and then the cell-seeded scaffolds were rinsed with PBS and fixed in glutaraldehyde $2.5 \%$ for $1 \mathrm{~h}$. For dehydration, the scaffolds were put into some gradient alcohol concentration and then dried; and examined using SEM.

\section{Collection of umbilical cord blood}

HSC were obtained from fresh CB-derived CD34+ progenitors. UCB samples were collected from healthy, full-term deliveries after written mother's informed consent according to institutional guidelines, transferred to the laboratory at $4{ }^{\circ} \mathrm{C}$, and processed within $4 \mathrm{~h}$.

\section{Isolation of CB CD34+ cells}

$C B$ was diluted in 1:5 with hydroxyl ethyl starch solution (HES, Grifols, Barcelona, Spain), and mononuclear cells were separated from the cord blood by density gradient centrifugation. In the following, CD34+ cells were isolated from the mononuclear cells using mini-magnetically activated cell sorting (MACS) technique as per manufacturer's instructions. The purity of the separated cells was analyzed by flow cytometry.

\section{Ex vivo expansion of CD34+ cells in serum-free medium}

CD34+ cells were seeded at a density of $1 \times 10^{4}$ cells/ well/250 $\mu$ l of Stemline II medium (Sigma, Darmstadt, Germany), in 24 well plates drop by drop. Added as a supplement SCF $(50 \mathrm{ng} / \mathrm{ml}$, Peprotech, London, UK), TPO (50 ng/ml, Gibco, Gran Island, NY) and FLT-3 (50 ng/ $\mathrm{ml}$, Gibco, Gran Island, NY). Seeded scaffolds incubated in a humidified atmosphere at $37^{\circ} \mathrm{C}$ with $20 \% \mathrm{O}_{2}$ and $5 \% \mathrm{CO}_{2}$ for 10 days. Every $48 \mathrm{~h}$ half of the medium was replaced with fresh medium and cells were counted. For performed test, removed cells on the scaffold by sampler and washed scaffold with medium for gained more cells. Then, centrifuged cells with medium for remove debris and washed by PBS. Tissue culture polystyrene (TCPS) was used as a control condition. 
Table 1: Sequences of Primers for real time polymerase chain reaction analysis.

\begin{tabular}{|c|c|c|c|c|}
\hline Gene name & Forward (5'-3') & Reverse (5'-3') & $\begin{array}{l}\text { Size band } \\
\text { (bp) }\end{array}$ & $\begin{array}{l}\text { Annealing } \\
\text { Temperature }\left({ }^{\circ} \mathrm{C}\right)\end{array}$ \\
\hline GAPDH & TGCACCACCAACTGCTTAGC & GGCATGGACTGTGGTCATGAG & 86 & 60 \\
\hline BMI-1 & GAAGATAGAGGAGAGGTTGCAG & CCGATCCAATCTGTTCTGGT & 101 & 60 \\
\hline hTERT & CGGAAGAGTGTCTGGAGCAA & GGATGAAGCGGAGTCTGGA & 144 & 60 \\
\hline HOXA9 & GCGCCTTCTCTGAAAACAAT & CAGTTCCAGGGTCTGGTGTT & 145 & 62 \\
\hline HOXB4 & CCTGGATGCGCAAAGTTCA & ААТTССТTСТССАGСТCСAАGA & 114 & 64 \\
\hline CXCR4 & TGAACCCCATCСTCTATGCTT & GATGAATGTCCACCTCGCTTT & 126 & 64 \\
\hline LFA-1 & GAGGACAACTTGTACAAGAGGA & CTCGGTGAGTTTCTCGTAGG & 140 & 60 \\
\hline VLA-4 & ATGTTGCGCATGTTCTACTG & AGCCTTCCACATAACATATGAG & 133 & 60 \\
\hline VLA-5 & CAGATCCTCAGCAAGAATCTC & CGTAACTCTGGTCACATATAGG & 299 & 60 \\
\hline
\end{tabular}

\section{Flow cytometry analysis}

Phenotypic analyses of the expanded cells were performed on the $10^{\text {th }}$ day for evaluation the capability of HSC expansion and differentiation. The expanded cells in control and 3D culture medium were collected and stained with monoclonal antibody CD34-PE and CD45FITC, lymphoid markers (CD2-FITC, CD3- FITC and CD19FITC), myeloid markers (CD13-FITC and CD33- FITC) and monocyte (CD14-FITC) (Dako, Glostrup, Denmark) against human epitope. After the addition of antibodies (10 $\mu \mathrm{l}$ with 1:10 dilution), the cells were incubated for $30 \mathrm{~min}$ at $4^{\circ} \mathrm{C}$. Excess antibody was removed by washing with PBS and kept at $4{ }^{\circ} \mathrm{C}$ till acquiring. Appropriate isotype control was used to set compensation and confirm the specificity. A minimum of 10,000 events was for each sample on a Partec PAS (Munster, Germany) flow cytometer. Flow Jo software was used for data analysis (Tree Star, Inc., Ashland, OR).

\section{RNA isolation and Real-time PCR analysis}

Total RNA was isolated using Trizol (Invitrogen, Carlsbad, CA) from purified progenitor cells and ex vivo expanded cells as per manufacturer's instructions. The isolated RNA was quantitated using a spectrophotometer. First strand cDNA synthesis was carried out by using random hexamer primers and the Superscript II reverse transcriptase kit (Invitrogen, Carlsbad, CA) according to manufacturer's instructions.

The real-time PCR was performed using the SYBRGreen I PCR master mix for evaluating the expression levels of homing (CXCR4, VLA-4, VLA-5, and LFA-1), self renewality (BMI-1, hTERT, HOXA9, and HOXB-4) and the GAPDH reference gene. Briefly, PCR was performed in a $12.5 \mu \mathrm{l}$ total volume containing $1 \mu \mathrm{l}$ of cDNA, $6.25 \mu \mathrm{l}$ of $2 \mathrm{X}$ SYBR Green mix (Amplicon, Aalborg, Denmark), and 10 $\mu \mathrm{mol} / \mathrm{l}$ primer pairs. After an initial denaturation at the $95{ }^{\circ} \mathrm{C}$ for $15 \mathrm{~min}$, following procedure were performed using $A B I$ step one PCR cycler ( $A B I$, Vernon, $C A): 20 \mathrm{~s}$ at the $95^{\circ} \mathrm{C}, 60 \mathrm{~s}$ at the $60^{\circ} \mathrm{C}$ for GAPDH, LFA-1, VLA-4, VLA-5, hTERT and BMI-1, and $62{ }^{\circ} \mathrm{C}$ for HOX-A9, and 64 ${ }^{\circ} \mathrm{C}$ for $C X C R-4$ and $H O X-B 4$ for 40 cycles. Samples without reverse transcription were used as negative controls. The data are presented as the relative expression of the genes of interest relative to the internal control gene as determined by the $2^{(-\Delta \Delta C T)}$ method. The primers used for real-time PCR for all gene amplifications are shown in Table 1.

\section{Colony forming unit assay}

The freshly isolated CB hematopoietic stem/progenitor cells expanded in culture on day 10 were incubated in $2 \mathrm{ml}$ methylcellulose culture medium ( $\mathrm{H} 4435$, Stem Cell Technology, Vancouver, Canada) plated in six-well dishes at a concentration of $1 \times 10^{4}$. All cultures were carried out in triplicate, incubated at $37{ }^{\circ} \mathrm{C}$ and $5 \% \mathrm{CO}_{2}$ humidified atmosphere in the air, and after 14 days of culture, colony forming unit of granulocyte-erythrocyte-monocyte-megakaryocyte colony forming units (CFU-GEMM), granulocyte-macrophage colony forming units (CFU-GM) and erythroid burst-forming units (BFU-E) were scored under an inverted microscope.

\section{Statistical analysis}

The parametric ANOVA test was used in order to compare the number and expansion of the total nucleated cells, the percentage, and expansion of CD34p cells as well as the number of colonies in the four studied groups. GraphPad Prism 6.0 software (GraphPad Software, La Jolla, CA) used for analysis. The values were plotted as mean \pm standard deviation Probability value: ${ }^{*} p<0.05,{ }^{* *} p<0.01,{ }^{* * *} p<0.001$ and ${ }^{* * * *} p<0.0001$ were considered statistically significant.

\section{Results}

\section{Electrospun scaffolds characterization}

Once the polymer solution was electrospun, $\mathrm{PCL}$ nanofibers showed an average thickness and fiber diameters $0.3 \mathrm{~mm}$ and $10 \mathrm{~mm}$ respectively. To study microscopic scaffold architecture, each specimen was imaged by SEM.

Better kept of CD34+ cells expansion in PCL scaffold coated with collagen

The average $7 \times 10^{5} / \mathrm{ml} \mathrm{CD34+} \mathrm{cells} \mathrm{from} \mathrm{each} \mathrm{cord}$ blood unit gained with the mini-MACS system. The purity of isolated CD34+ cells was $85.4 \% \pm 7.6 \%$ Figure 1. In overall, the enormous of cells were spherical cells that were nonadherent to plastic.

Figure 2 showed the 3D culture system provided a 

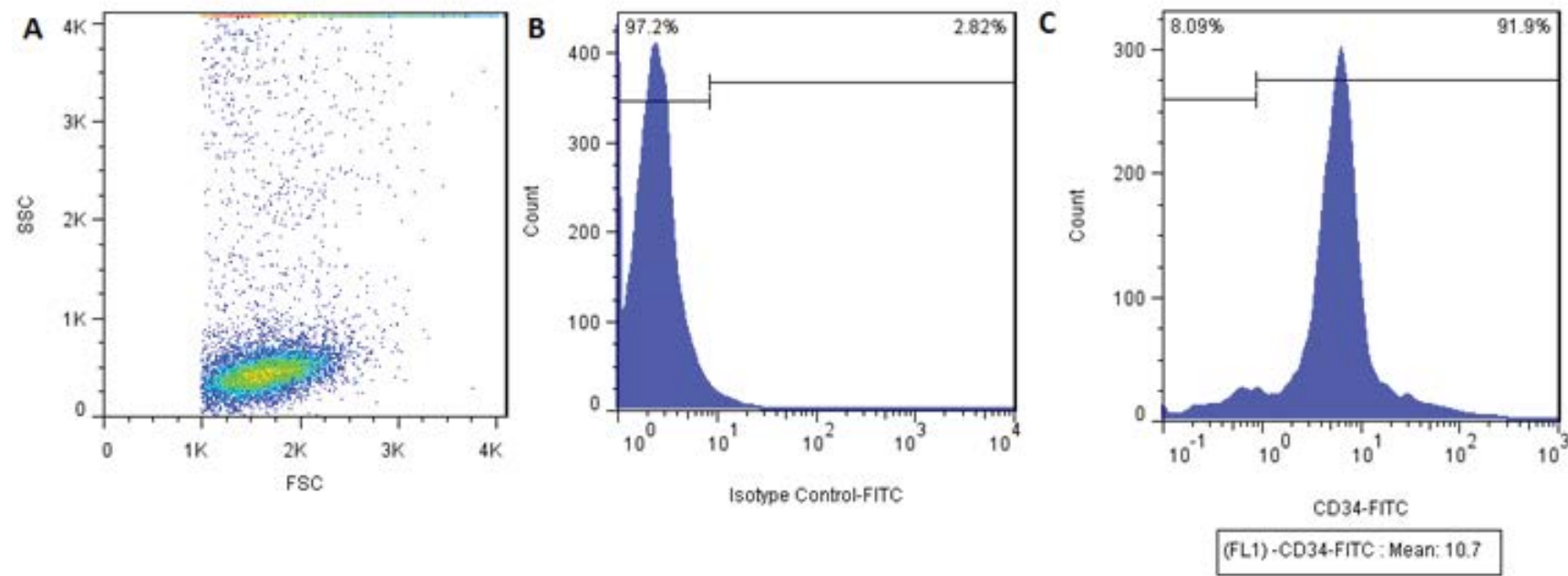

Figure 1: Flowgram of CD34+ cord blood cells purity percentage. A) Distribution of cells by size and granularity; B) Control isotype; C) CD34+ cell distribution after purification by the MidiMACS column.
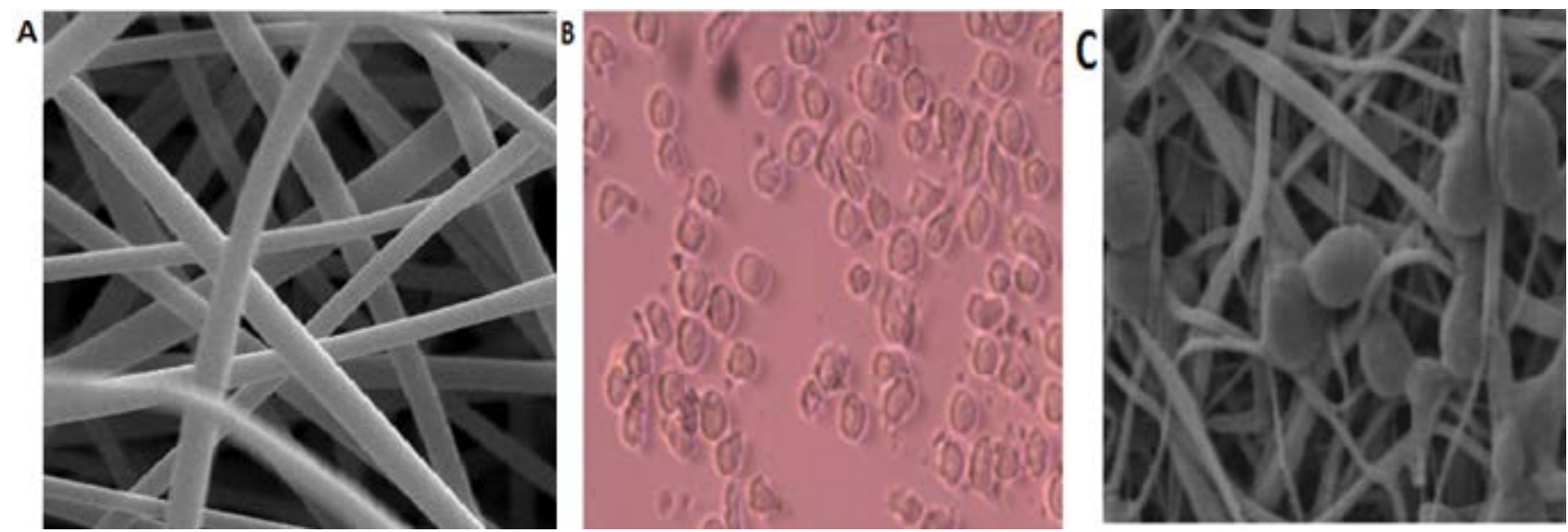

Figure 2: Images of light and electronic microscopes. A) Scanning electron micrograph of polycaprolactone nano-fibers; B) CD34+ CB cells cultured in 2D medium and; C) CD34+ CB cells cultured in PCL nano-fiber scaffold coated with Collagen after 10 days.

superior number of CD34+ cells when compared with conventional 2D cultures. Average fold increase of the TNCs after 10 days culture in the PCL coated with collagen (3D) and traditional static (2D) culture systems were $50 \pm 1.63$ and $38.3 \pm 3.3$, respectively Figure 3 .

\section{Augmented myeloid markers in cells expanded in PCL scaffold coated with collagen}

The frequency of CD34+ cells before the expansion was $85.4 \pm 7.6$ and after 10 days culture in the TCPS dropped to $5.75 \% \pm 1.25 \%(p<0.001)$. Cells expanded in collagen-coated PCL scaffold better kept the frequency of CD34+ cells and this value was $40.6 \% \pm 1.8 \%$ ( $p<$ $0.001)$. While nucleated cells in the $3 \mathrm{D}$ and $2 \mathrm{D}$ culture system proliferated $50 \pm 1.63$ and $38.3 \pm 3.3$ times, respectively, the absolute number of CD34+ cells in the $3 \mathrm{D}$ and $2 \mathrm{D}$ culture systems increased up to roughly 20 and 2.66 times the initial cell number, respectively $(p<$ 0.001 ) Figure 3 . The frequency of the lymphoid surface markers (CD2+ and CD3+ for T cells and CD19+ for B cells) in cells expanded in collagen-coated scaffold was lower than TCPS culture. Remarkably, frequency of CD13+ and
CD33+ (committed granulocyte-monocyte progenitor markers) and CD14+ (mature monocytes marker) and CD45+ surface markers in collagen-scaffold were higher than the TCPS system Figure 4.

Boosted homing and selfrenewality of the cells expanded in the PCL-collagen scaffold

The levels of expression of CXCR-4, LFA-1, VLA-4, VLA-5 and BMI-1, hTERT, HOXA9 and HOXB4 evaluated for homing and selfrenewality of expanded CD34+ cells, respectively. The CD34+ cells expanded in collagencoated PCL scaffold revealed superior levels of homing genes than unexpanded CD34+ cells except for VLA4. Compared with that cultured in the $2 D$ cell culture system, CD34+ cells expanded in collagen-coated PCL scaffold displayed increased expression of homing genes, while VLA-4 (7.1-fold) variation was statistically significant $(p=0.0001)$. Expanded CD34+ cells in the 2D cell culture system diminished expression of homing genes compared to before expansion that decreased expression of VLA-4 which was statistically significant ( $p$ $=0.0001$ ) and only VLA -5 had somewhat increased. 
Average fold changes of self-renewal genes in 3D scaffold system compared to unexpanded CD34+ cells was higher and this increase for BMI-1 (19.98-fold) and $h T E R T$ (40.7-fold) was statistically significant ( $p$ $=0.0001)$. Compared to before expansion the $2 \mathrm{D}$ cell culture system had not statistically significant increase

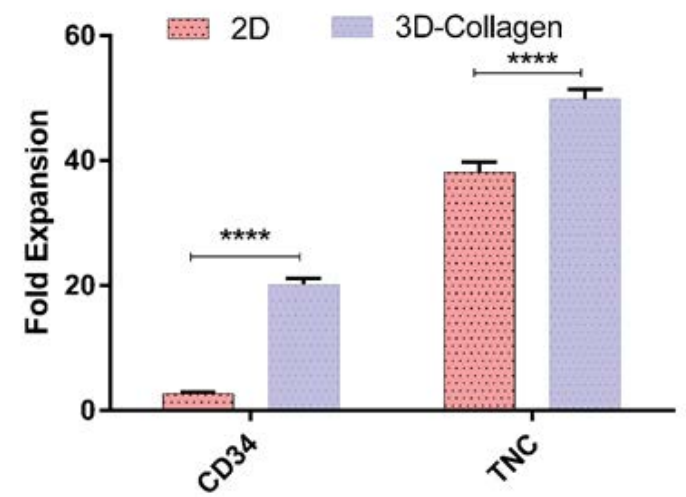

Figure 3: Fold expansion of CD34+ cells and TNC in 2D and $3 D$ medium. The significant increase of CD34+ cells and TNC saw after 10 days culture of 10000 CB HSPCs in $\mathrm{PCL}$ nanofibers than on conventional culture.

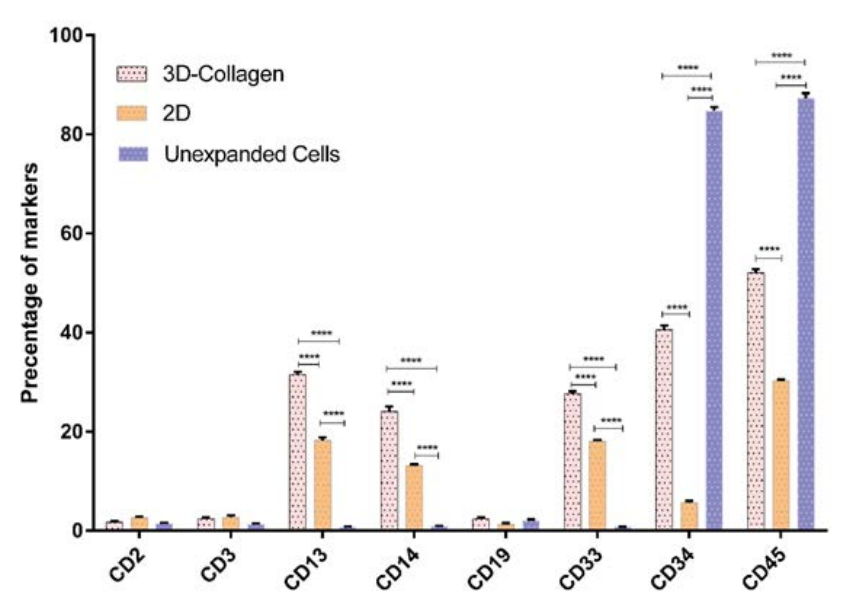

Figure 4: Frequency of surface linage markers compared among unexpanded cells, 2D, and 3D cells after 10 days culture. Hematopoietic stem cell (CD34+), pan-leukocyte (CD45), granulocytes (CD13 and CD33), lymphocytes (CD2, CD3, and CD19) and monocyte (CD14) markers in unexpanded cells, 2D and 3D cell culture systems after 10 days expansion. in self renewality genes. 3D scaffold comparisons to 2D cell culture system exhibited increased expression of self renewality genes and expression of BMI-1 (5.7-fold) and $h T E R T$ (13.7-fold) was statistically significant $(\mathrm{p}=$ 0.0001) Figure 5.

\section{Enhanced colony formation of the cells expanded in the PCL-collagen scaffold}

As an initial assessment of function, we determined the frequency of colonies in cells recovered from the culture systems using conventional colony-forming unit (CFU) assays Figure 6. We observed a 1.8-fold increase in CFU-GEMM number, a 1.38-fold increase of BFU-E/ CFU-E number, a 1.17-fold increase in total CFU in collagen-coated PCL scaffold compared with the 2D cell culture system. The number of CFU-GM was nearly equal.
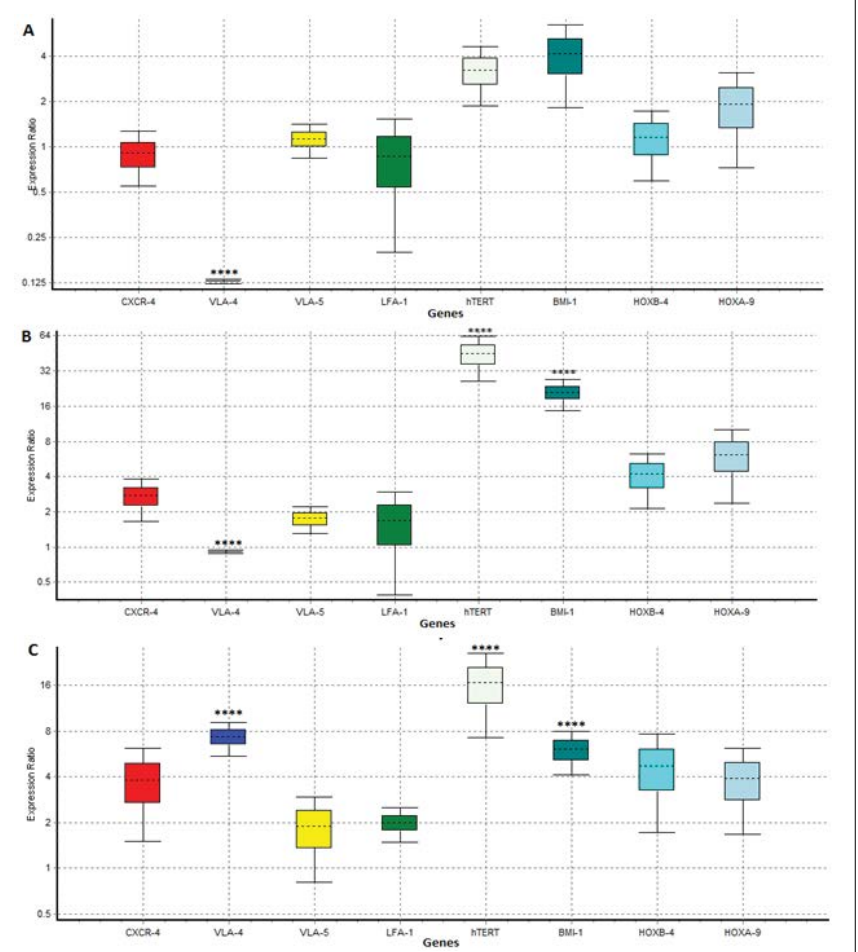

Figure 5: Relative fold changes of homing and self-renewal genes after 10 days culture. A) 2D culture system compared to unexpanded cells; B) 3D cell culture systems compared to unexpanded cells; C) 3D cell culture systems compared to $2 \mathrm{D}$ culture system.

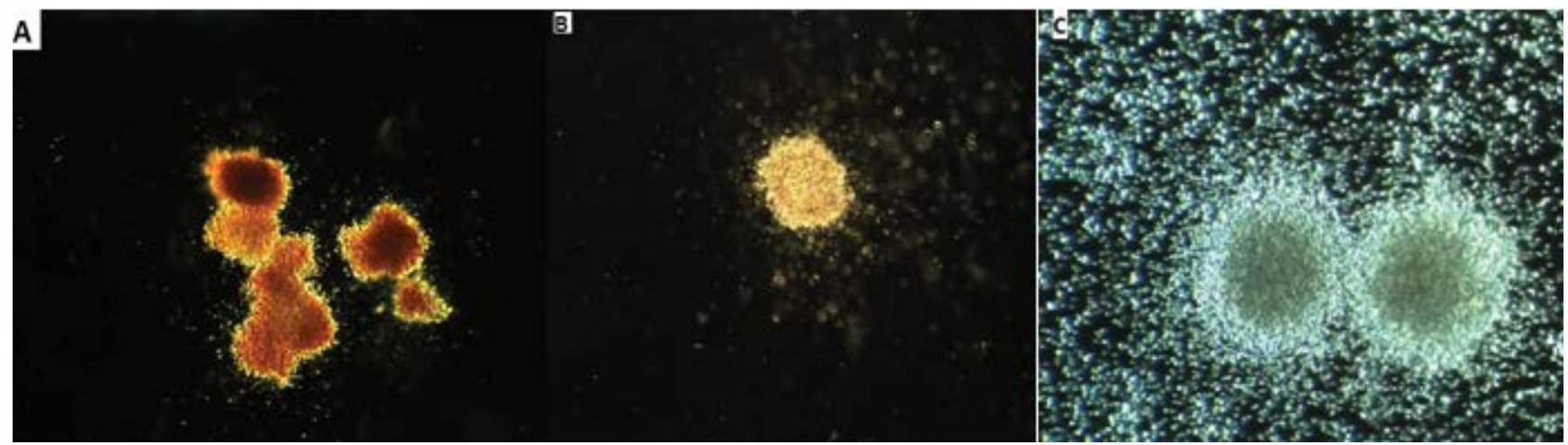

Figure 6: Image of BFU-E/CFU-E A), CFU-GM B) and CFU-GEMM C) based on total CFU analyzed for cells expanded in different culture system for 10 days. 


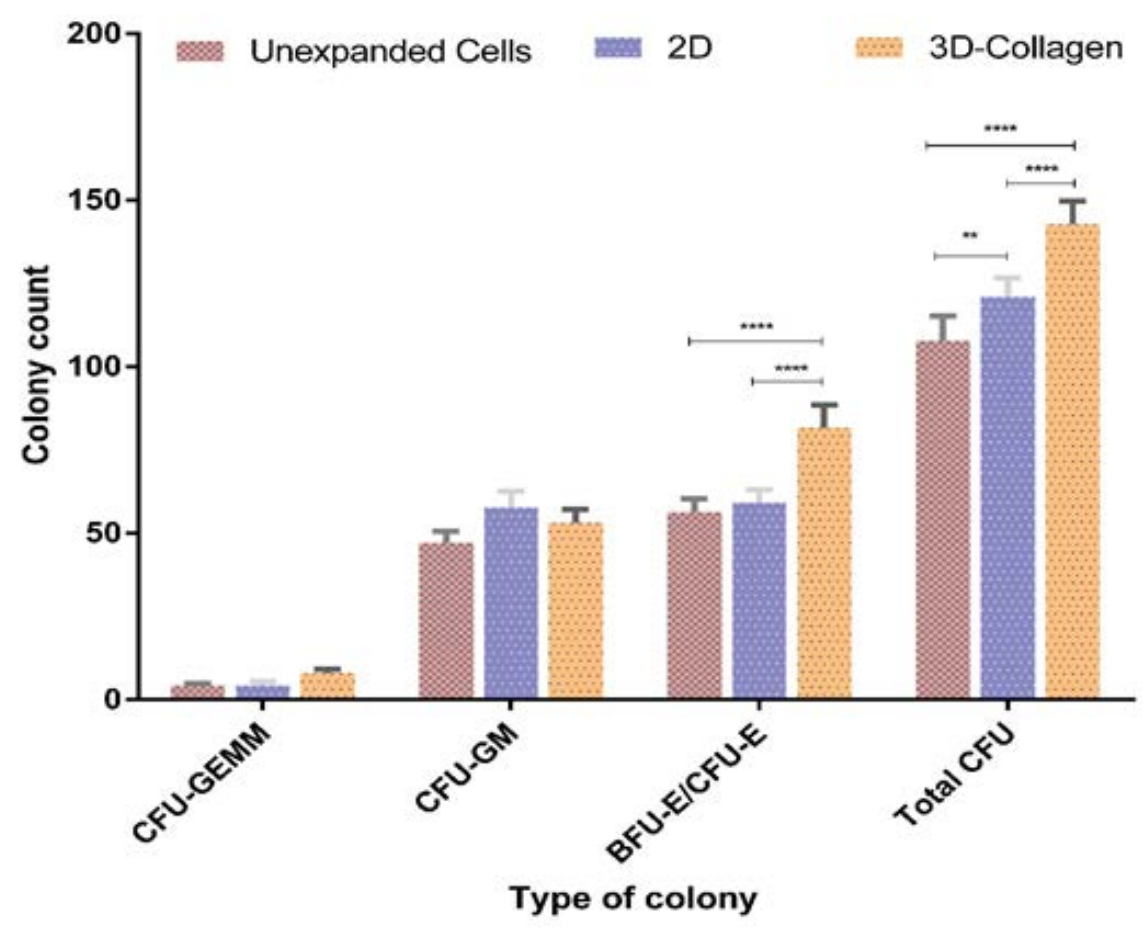

Figure 7: Colony formation assay. Colony count in unexpanded cells, 2D and 3D cell culture systems after 10 days culture.

The proportions of CFU-GM, BFU-E/CFU-E, CFUGM and CFU-GEMM in total CFU number are plotted in Figure 7. Compared with unexpanded CD34+ cells, the collagen-coated scaffold preserved a comparable percentage of CFUGEMM ( $5 \%$ vs. $4 \%$ ) for unexpanded cells ( $p>0.05)$, a $8 \%$ increase of BFUE/CFU-E $(57.5 \%$ vs. $49.5 \%)(p=0.001)$ and a $6 \%$ increase of CFU-GM $(43 \%$ vs. $37 \%)(p=0.001)$.

\section{Discussion}

Ex vivo expansion of CB HSCs is a great strategy for an improvement in the clinical outcome of HSCT. In this study, we have developed a 3D PCL nanofibrous scaffold coated with collagen for ex vivo expansion of cord blood-derived CD34+ cells. This scaffold provided an appropriate substrate for cell entrapment, 3D cellcell interaction, cell-ECM interactions, and proliferation. The culture system revealed improved maintenance and expansion of HSCs within 3D scaffolds that mimic the bone marrow microenvironment. In the previous study showed that $\mathrm{PCL}$ as promising alternatives to a traditional system for the culture of CB-derived CD34+ progenitors. Polycaprolactone $(\mathrm{PCL})$ is a biocompatible, biodegradable and nontoxic linear aliphatic polyester and it can be easily fabricated into thin membranes while maintaining its mechanical strength. Because of the characteristics described above, PCL has been used in various applications, including scaffolds, nanoparticles, nanofibers, composites, hydrogels and films for TE [14].

Also, confirmed that the bone marrow ECM proteins (including collagens, fibronectin, and laminin) prepare anchorage sites that are recognized by cells through specific cell adhesion molecules. For this reason, the ECM plays a critical function in the maintenance of stem cells in their niches as well as their mobilization from the niche $[11,13]$. Thus, the superiority of 3D PCL nanofiber scaffolds coated with collagen, compared to 2D cultures, expanding HSC, is predominantly based on the scaffold architecture and ECM protein.

Quantitatively, the addition of collagen in our 3D culture methods was found statistically significant compared with the 2D method. We have shown that adding the growth factors cocktail (TPO, Flt-3, and SCF) to the culture medium amplified number of CD34+ cells and TNCs to 2.66-fold and 38-fold, respectively. In contrast, after 10 days of expansion in a 3D PCL-collagen culture medium, we detected 20 -fold increases in the number of CD34+ progenitors and 50-fold in the number of TNCs.

Our electrospun fiber scaffolds are their microscale construction with greater surface-to-volume ratio and capacity to selectively enhance absorption of ECM proteins that are able to regulate communications of the cells with the microenvironment and cell interaction [14]. In 2D cell culture system, this communication capacity is low and restricted to neighboring cells, therefore, TNC and CD34+ cell expansion in 2D is lower than 3D.

In the previous study, we have shown that $\mathrm{PCL}$ nanofiber scaffold coated with fibronectin had a higher number of CD34+ cells and TNCs number than traditional culture medium [15]. In 2016, Kang, et al. revealed that the culture of $\mathrm{CB}$ derived $\mathrm{CD} 34+$ in $\mathrm{PCL}$ coated with fibronectin and collagen had a greater number of TNC and CD34+ cells compared to 2D culture system [16].

Investigation of amplification of CD34+ cells with a CFC assay showed greater colony forming capability of cells cultured in the 3D PCL-Collagen scaffold than 2D 
method. In particular, a statistically significant difference between the 2 tested culture conditions was detected in BFU-E and total CFU cells after day 10 of culture. This finding suggested that $3 \mathrm{D}$ culture allows maintenance of hematopoietic cell quality for a longer time.

Previous studies have confirmed that 3D scaffolds could support the better maintenance of HSCs. However, if use MSCs or ECM protein as feeder layer, their consequences could be improved, because of cellcell interaction between CD34+ cells and feeder cells or ECM [16-21].

The frequency of cells that expressed specific markers were also evaluated by flow cytometry on days 10 and 3D PCL-collagen scaffold demonstrated a higher frequency of myeloid and lower lymphoid markers compared to the conventional culture medium. However, 3-D culturing systems tended to maintain phenotype to a greater degree than the TCPS group 2D culturing system. These results reveal that 3D PCLcollagen scaffold provides conditions desirable for ex vivo expansion, and phenotype maintenance of HSPCs. Previous studies support these results [17].

The importance of HOXB-4, BIM-1, HOX-A9, and hTERT in HSC self-renewal has been demonstrated before [15]. Several studies revealed that amplified expression of Bmi-1 and HOXB-4 directed to the symmetrical cell division of HSC and effects in HSC expansion [22,23]. Also, other studies exhibited an ectopic expression of HOXB-4 can mediate a significant expansion of HSC and also HOXB-4 and HOXA-9, enhance HSC activity [24].

Human telomerase reverse transcriptase ( $h T E R T$ ), in human $\mathrm{CB} C D 34+$ cells leads to an enhanced survival of mature hematopoietic cells [25]. Our results confirm this study and showed that after expansion, especially in 3D PCL nano-scaffold increased self-renewal genes.

Efficient homing of circulating HSC/HPCs into the bone marrow niche required at high levels for CXCR-4, $L F A-1, V L A-4$, and $V L A-5$. It has been confirmed that the close communication between CAMs receptors on HSPC and ECM in the bone marrow microenvironment plays a crucial role in stem cell homing to the extravascular bone marrow niche $[13,15]$.

The different chemistries of the scaffold, scaffold geometry (porosities, fiber diameters, and orientation), physicochemical parameters (hydrophobicity, degradation time or protein adsorption) have an influence on HSC expansion. Also, culture condition such as contact with stromal cells or ECM, the number of initiation of CD34+ cell culture, oxygen concentration, shear stress, duration of culture, cytokine cocktail, and cell culture medium has an impact on expansion and maintenance of HSC [14].

\section{Conclusions}

We have developed a biomimetic nanofiber PCL scaf- fold based co-cultures of CD34+ cells and collagen as the main ECM protein to mimic the natural microenvironment of HSCs. In comparison to the conventional CD34+ cell culture, nanofiber PCL scaffold coated with collagen could effectively promote the maintenance of CD34+ phenotype and primitive progenitor cells. Thus ex vivo expansion of CD34+ cells could be effectively promoted in PCL-based coating with collagen.

\section{Acknowledgments}

The authors are thankful to E Janzamin assisting in flow cytometery analysis. Authors acknowledge to Dr. M Zarrabi for help us for cord blood supply.

\section{Financial and Competing Interests Disclosure}

This work was supported by the Deputy of Research Affairs and Faculty of Medical Sciences [Grant No. 52/2767] at Tarbiat Modares University. The authors have no other relevant affiliations or financial involvement with any organization or entity with a financial interest in or financial conflict with the subject matter or materials discussed in the manuscript apart from those disclosed.

No writing assistance was utilized in the production of this manuscript.

\section{Ethical Conduct of Research}

The experimental protocol of the study was approved by the Ethics Committee of Tarbiat Modares University. The informed consent was obtained for the delivery of cord blood from pregnant mothers.

\section{References}

1. Gluckman E (2009) History of cord blood transplantation. Bone Marrow Transplant 44: 621-626.

2. Bari S, Seah KK, Poon Z, Cheung AM, Fan X, et al. (2015) Expansion and homing of umbilical cord blood hematopoietic stem and progenitor cells for clinical transplantation. Biol Blood Marrow Transplant 21: 1008-1019.

3. Chou S, Chu P, Hwang W, Lodish H (2010) Expansion of human cord blood hematopoietic stem cells for transplantation. Cell Stem Cell 7: 427-428.

4. Tung S, Parmar S, Robinson S, De Lima M, Shpall E (2010) Ex vivo expansion of umbilical cord blood for transplantation. Best Pract Res Clin Haematol 23: 245-257.

5. Stanevsky A, Goldstein G, Nagler A (2009) Umbilical cord blood transplantation: Pros, cons and beyond. Blood Rev 23: $199-204$.

6. Gluckman E, Rocha V (2009) Cord blood transplantation: State of the art. Haematologica 94: 451-454.

7. Dahlberg A, Delaney C, Bernstein ID (2011) Ex vivo expansion of human hematopoietic stem and progenitor cells. Blood 117: 6083-6090.

8. Flores-Guzmán P, Fernández-Sánchez V, Mayani H (2013) Concise review: Ex vivo expansion of cord blood-derived hematopoietic stem and progenitor cells: Basic principles, experimental approaches, and impact in regenerative medicine. Stem Cells Transl Med 2: 830-838. 
9. Baron F, Ruggeri A, Nagler A (2016) Methods of ex vivo expansion of human cord blood cells: Challenges, successes and clinical implications. Expert Rev Hematol 9: 297-314.

10. Adams GB, Scadden DT (2006) The hematopoietic stem cell in its place. Nat Immunol 7: 333-337.

11. Frantz C, Stewart KM, Weaver VM (2010) The extracellular matrix at a glance. J Cell Sci 123: 4195-4200.

12. Lee-Thedieck C, Spatz JP (2014) Biophysical regulation of hematopoietic stem cells. Biomaterials Science 2: 15481561.

13. Klamer S, Voermans C (2014) The role of novel and known extracellular matrix and adhesion molecules in the homeostatic and regenerative bone marrow microenvironment. Cell Adh Migr 8: 563-577.

14. Ferreira MSV, Mousavi SH (2018) Nanofiber technology in the ex vivo expansion of cord blood-derived hematopoietic stem cells. Nanomedicine 14: 1707-1718.

15. Mousavi SH, Abroun S, Soleimani M, Mowla SJ (2018) 3-Dimensional nano-fibre scaffold for ex vivo expansion of cord blood haematopoietic stem cells. Artif Cells Nanomed Biotechnol 46: 740-748.

16. Kang YG, Shin JW, Park SH, Kim YM, Gu SR, et al. (2016) A three-dimensional hierarchical scaffold fabricated by a combined rapid prototyping technique and electrospinning process to expand hematopoietic stem/progenitor cells. Biotechnol Lett 38: 175-181.

17. Mortera-Blanco T, Mantalaris A, Bismarck A, Aqel N, Panoskaltsis N (2011) Long-term cytokine-free expansion of cord blood mononuclear cells in three-dimensional scaffolds. Biomaterials 32: 9263-9270.
18. Ferreira MSV, Jahnen-Dechent $W$, Labude $N$, Bovi $M$, Hieronymus T, et al. (2012) Cord blood-hematopoietic stem cell expansion in 3D fibrin scaffolds with stromal support. Biomaterials 33: 6987-6997.

19. Ferreira MV, Labude $N$, Piroth $D$, Jahnen-Dechent $W$, Knüchel R, et al. (2012) Compatibility of different polymers for cord blood-derived hematopoietic progenitor cells. J Mater Sci Mater Med 23: 109-116.

20. Batnyam O, Shimizu H, Saito K, Ishida T, Suye S-i, et al. (2015) Biohybrid hematopoietic niche for expansion of hematopoietic stem/progenitor cells by using geometrically controlled fibrous layers. RSC Advances 5: 80357-80364.

21. Pan X, Sun Q, Zhang $Y$, Cai H, Gao $Y$, et al. (2017) Biomimetic macroporous pcl scaffolds for ex vivo expansion of cord blood-derived CD34+ cells with feeder cells support. Macromol Biosci 17: 1-12.

22. Antonchuk J, Sauvageau G, Humphries RK (2002) HOXB4induced expansion of adult hematopoietic stem cells ex vivo. Cell 109: 39-45.

23. Iwama $A$, Oguro $H$, Negishi $M$, Kato $Y$, Morita $Y$, et al. (2004) Enhanced self-renewal of hematopoietic stem cells mediated by the polycomb gene product Bmi-1. Immunity 21: 843-851.

24. Amsellem S, Pflumio F, Bardinet D, Izac B, Charneau P, et al. (2003) Ex vivo expansion of human hematopoietic stem cells by direct delivery of the HOXB4 homeoprotein. Nat Med 9: 1423-1427.

25. Elwood N (2004) Telomere biology of human hematopoietic stem cells. Cancer Control 11: 77-85. 